\title{
Follow-up of X-ray transients detected by SWIFT with COLORES using the BOOTES network
}

\section{D. Caballero-Garcia ${ }^{a}$, M. Jelínek ${ }^{b}$, A. J. Castro-Tirado ${ }^{b c}$, R. Hudec $^{a d}$, R. Cunniffe $^{b}$, O. Rabaza ${ }^{e}$ and L. Sabau-Graziati ${ }^{f}$}

${ }^{a}$ Czech Technical University in Prague, Faculty of Electrical Engineering

Technická 2, 16627 Praha 6 (Prague), Czech Republic

E-mail: cabalmalefel.cvut.cz

${ }^{b}$ Instituto de Astrofísica de Andalucía (IAA-CSIC)

P.O. Box 03004, E-18080, Granada, Spain

${ }^{c}$ Unidad Asociada Departamento de Ingeniería de Sistemas y Automática, E.T.S. de Ingenieros Industriales, Universidad de Málaga

Málaga, Spain

${ }^{d}$ Astronomical Institute, Academy of Sciences of the Czech Republic

25165 Ondřejov, Czech Republic

e Área de Ingeniería Eléctrica, Dpto. de Ingeniería Civil, Univ. de Granada

Granada, Spain

${ }^{f}$ División de Ciencias del Espacio (INTA)

Torrejón de Ardoz, Madrid, Spain

\begin{abstract}
The Burst Observer and Optical Transient Exploring System (BOOTES) is a network of telescopes that allows the continuous monitoring of transient astrophysical sources. It was originally devoted to the study of the optical emission from gamma-ray bursts (GRBs) that occur in the Universe. In this paper we show the initial results obtained using the spectrograph COLORES (mounted on BOOTES-2), when observing compact objects of diverse nature.
\end{abstract}

Swift: 10 Years of Discovery,

2-5 December 2014

La Sapienza University, Rome, Italy 


\section{Introduction}

Compact objects result from the end points of massive stars. When the burning of the star runs out and if the star is massive enough (superseding certain stability limits) then collapse occurs. In the case of stars with a core mass of $<1.4 \mathrm{M}_{\odot}$ a white dwarf (WD) of electron-degenerate matter is formed that, if not exceeding this limit, will be radiating energy (and cooling down) in a (more or less) stable way for $\geq 10^{9} \mathrm{Gyr}$. In the case of more massive stars, the evolution is more complicated, since the core of electron-degenerate-matter is not able to sustain gravitational forces and collapse occurs. Gravitational sources can stop the fatal end only for stars with a core $<3 \mathrm{M}_{\odot}$, thus forming a neutron star (NS), i.e. core of neutron-degenerated matter. Above this mass limit, there is no force in Nature able to stop the gravitational collapse and a black-hole (BH) is formed.

The astrophysical study of compact objects is only possible due to the emission of their associated accretion (either in the form of discs or streams of matter - the latter in the case of the polar cataclysmic variables) in X-rays. For this accretion disc to exist either a donor star (in the case of binary systems) or material (in the case of Active Galactic Nuclei - AGN) has to be present. In principle, an isolated compact object can not emit detectable radiation by current instrumentation. Nevertheless, we also refer to the recent advances that have shown that isolated neutron stars (the so called "magnetars") emit huge amounts of hard X-rays and/or $\gamma$-rays detected by current instrumentation ([1]). Therefore, observations in the X-ray regime of compact objects constitute the most important tool in order to understand the physical properties of matter in the very inner regions around them. Although the classification of X-ray emitting compact objects is rather complex ([2]) we will be referring here to three basic types: 1) cataclysmic variables; 2) accreting pulsars and 3) black hole X-ray binaries (i.e. composed by a WD, NS and BH plus a donor star for the cases 1), 2) and 3), respectively).

The X-ray emission from compact objects is highly variable (due to the small-size scales of the emitting regions involved). The main goal of the X-ray Swift satellite ([3]) is the detection of Gamma-Ray Bursts (GRBs) due to its high angular field of view and rapid (time) reaction. Nevertheless, it has also been revealed to be a fantastic tool for the study of the X-ray emission from compact objects (with often an unpredictable emission behaviour). Observations in the optical are performed by big and medium-sized telescopes on Earth. The former are not suitable for performing the rapid follow-up needed for the study of optical transients (as we will explain hereafter). These transients events are typically of short duration (from fractions of a second to a few days), because the physical processes that originate them are of limited duration/spatial extent. Robotic smaller telescopes are very well suited for performing such studies. This is due to several factors: their observing flexibility, their rapid response and slew times and the fact that they can be located worldwide working remotely (therefore allowing continuous monitoring). Of course, additional observations might be triggered after the transient has been detected with large X-ray/Optical Observatories. In this way we can perform deep studies on the nature of these sources.

\subsection{The Burst Optical Observer and Transient Exploring System and its Spectrographs}

BOOTES (acronym of the Burst Observer and Optical Transient Exploring System) is a worldwide network of robotic telescopes. It was originally designed from a Spanish-Czech collaboration that started in 1998 ([4, 5]). The telescopes are located at Huelva (BOOTES-1), Málaga (BOOTES- 
2), Granada, Auckland (BOOTES-3) and Yunnan (BOOTES-4), located at Spain, New Zealand and China, respectively. There are plans of extending this network even further (Mexico, South Africa, Chile,...). These telescopes are medium-sized $(\mathrm{D}=30-60 \mathrm{~cm})$, autonomous and very versatile. They are very well suited for the continuous study of the fast variability from sources of astrophysical origin (GRBs and Optical Transients - hereafter OTs - ).

Currently two spectrographs are built and working properly in the network at Málaga and Granada (in the optical and infra-red, respectively). In Sec. 2 we will show preliminary results obtained so far with COLORES at BOOTES-2 in the field of compact objects.

\subsection{COLORES}

COLORES stands for Compact Low Resolution Spectrograph ([6]). It is a spectrograph designed to be lightweight enough to be carried by the high-speed robotic telescope $60 \mathrm{~cm}$ (BOOTES$2)$. It works in the wavelength range of $(3800-11500) \AA$ and has a spectral resolution of $(15-$ 60) $\AA$. The primary scientific target of the spectrograph is a prompt GRB follow-up, particularly the estimation of redshift.

COLORES is a multi-mode instrument that can switch from imaging a field (target selection and precise pointing) to spectroscopy by rotating wheel-mounted grisms, slits and filters. The filters and the grisms (only one is mounted at the moment) are located in standard filter wheels and the optical design is comprised of a four-element refractive collimator and an identical fourelement refractive camera. As a spectroscope, the instrument can use different slits to match the atmospheric seeing, and different grisms in order to select the spectral resolution according to the need of the observation.

The current detector is a $1024 \times 1024$ pixels device, with 13 micron pixels. The telescope is a rapid and lightweight design, and a low instrument weight was a significant constraint in the design as well as the need to be automatic and autonomous. For further details on description, operation and working with COLORES we refer to M. Jelinek $\mathrm{PhD}$ thesis ([7] and references therein).

\section{Scientific results obtained using BOOTES-2/COLORES}

In the following we present some important scientific results obtained using BOOTES-2 and its low-resolution spectrograph COLORES, recently obtained (we refer to [8] for a summary of previously obtained results in the field of OTs).

\subsection{The accreting pulsar A $0535+262$}

The evolution of the $\mathrm{H}_{\alpha}$ equivalent width (EW) of the Be/X-ray binary system A $0535+262$ (also called HD 245770) was reported ([9]), using observations performed with the spectrograph COLORES at the 0.6 m telescope BOOTES-2 (Málaga, Spain) on 2015-01-27 at 22:05:31.736 UTC (MJD 57049.920), and with the spectrograph located at the $51 \mathrm{~cm}$ telescope of the Observatorio de Aras de los Olmos of the University of Valencia on 2015-01-29 at 01:00:00 UTC (MJD 57051.042).

The results indicate that the $\mathrm{H}_{\alpha}$ line (in emission) had an EW of the order of $(-6.6 \pm 0.9) \AA$ (MJD 57049.920) and (-7.9 \pm 0.2$) \AA$ (MJD 57051.042). This means that the size of the Be disk had decreased to half of the value measured on 2014 March 19 ([10]), being similar to the estimate during quiescence on 2012 March. Recently, this system showed a weak enhancement in the 


\section{A $0535+26$}

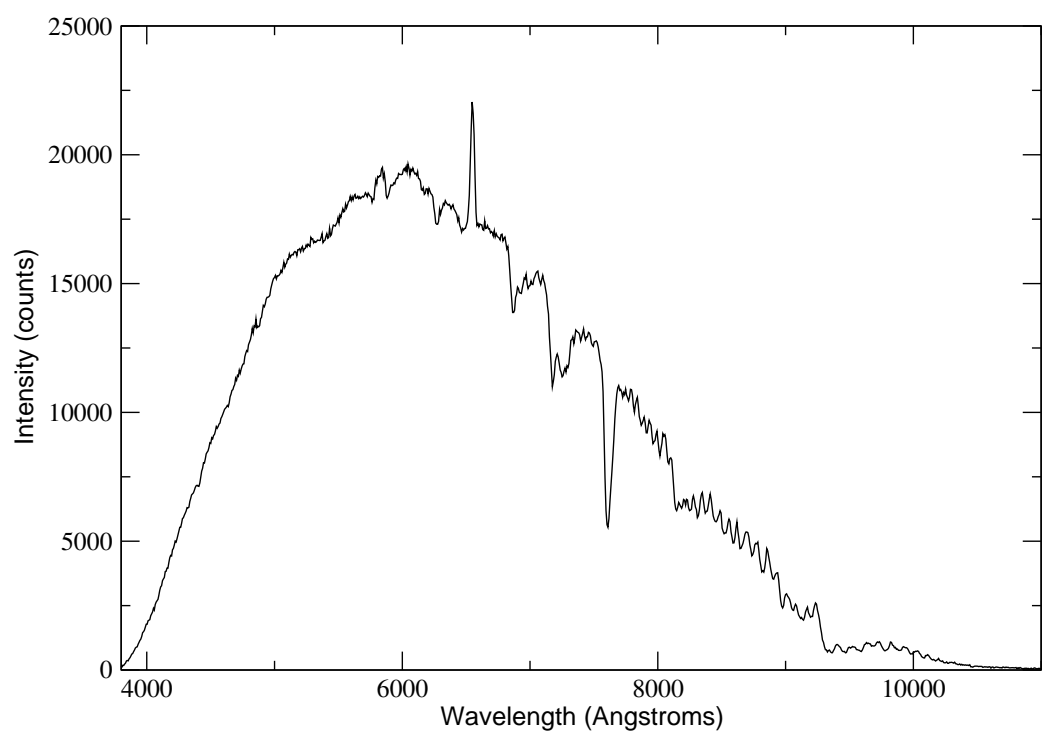

Figure 1: Optical spectrum in the range (3800-11000) from A 0535+26, obtained with BOOTES2/COLORES on $16^{\text {th }}$ Oct. (2014) at 01:37 UT.

optical luminosity ([11]), and a weak reawakening of X-ray pulsar's activity was thus expected. MAXI detected this source on 2015 January 29 ([12]), and the Swift/BAT monitoring web site was detecting its rise with a flux of $570 \pm 20 \mathrm{mCrab}$ (MJD 57057).

The behavior of this system might resemble the giant outburst of 2011 in which the EW of the $\mathrm{H}_{\alpha}$ line was initially of the order of $-9.5 \AA$ ([13]).

\subsection{The accreting black hole SS 433}

In light of the recent extreme outburst of SS 433 ([14, 15]) and follow-up by Swift ([16]) the $0.6 \mathrm{~m}$ TELMA robotic telescope at the BOOTES-2 (+COLORES) astronomical station in Málaga (Spain), obtained optical (4000 - 9000) A spectra starting at 2014-08-01 and ending at 2014-0807 , as reported ([17]). The most prominent feature of the spectra was the variable $\mathrm{H}_{\alpha}$ line. There was also a variable emission line at $7100 \AA$ that might correspond to a $300 \AA$ shifted $\mathrm{H}_{\alpha}$ P-fund line (7460 $\mathrm{A})$, previously reported in the literature ([18]).

\section{Discussion and conclusions}

The era of OTs is about to start. Since the beginning of the modern times the big telescopes have been the only resource for astronomers to study astrophysical sources. In spite of constituting the best tool for deep studies of individual targets, they are not properly suited for the discovery of optical transient sources. Their big size limits the speed they can cover the entire sky and the time for overheads might be longer than that for medium-sized telescopes. Many factors make them difficult to fully automatize and indeed currently none is completely robotic and autonomous. 
SS 433

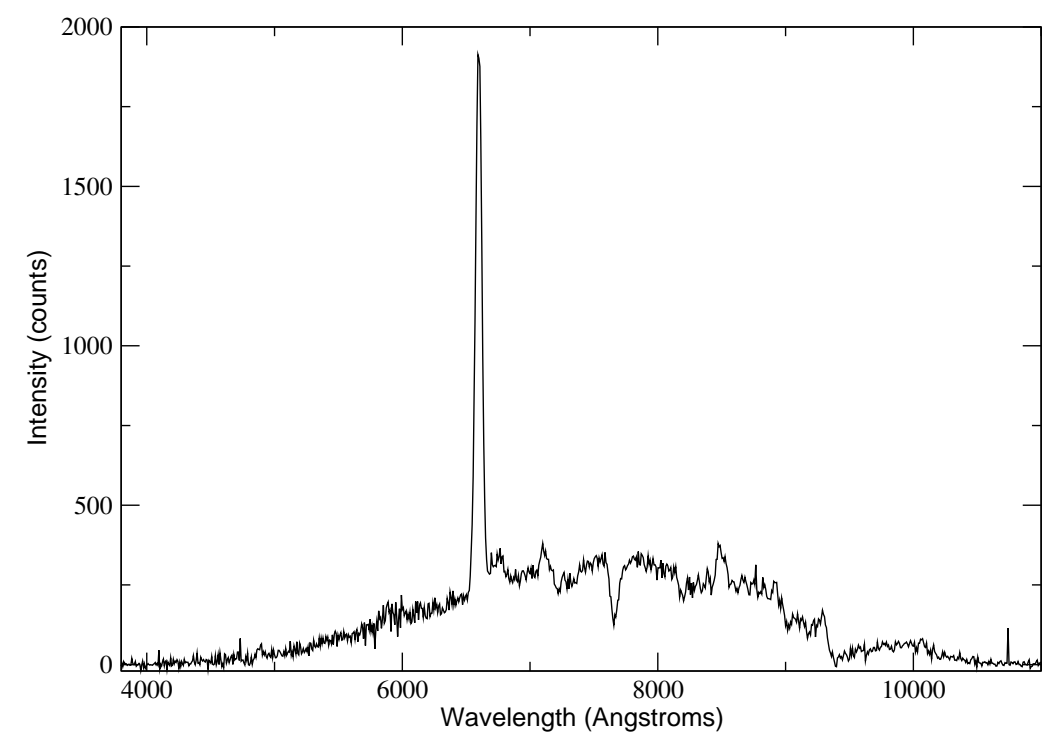

Figure 2: Optical spectrum in the range (3 800-11 000) from SS 433, obtained with BOOTES-2/COLORES on $2^{\text {nd }}$ Aug. (2014) at 02:33 UT.

Medium-sized telescopes (i.e. $\mathrm{D} \leq 1 \mathrm{~m}$ ) can be much quicker moving from target to target and time overheads are usually very small. Therefore, robotic medium-sized telescopes are currently the best ones for the follow-up and studies of the long-term variability of the astrophysical transient sources.

BOOTES-2 constitutes one step forward with respect to other robotic telescopes. This is because it is one (of the very few) robotic telescopes with a spectrograph mounted on it. It has demonstrated to work properly and efficiently and in this paper we give a first glimpse on the results obtained from its observations of compact objects. Since compact objects are transient sources variable in the sky (i.e. OTs) the Swift satellite constitutes a precious tool for detecting and following them. When an OT has been detected by Swift at X-rays, an alert is created to the scientific community (often through an Astronomer Telegram or the Gamma-ray Coordinates Network). In such a case the BOOTES telescopes start the observation, re-observing the target every time it is visible during the following nights.

Apart from the intensive campaign of follow-up of GRBs performed by the BOOTES network $(\approx 100$ GRBs have been observed so far), BOOTES-2 and its spectrograph (COLORES) are also providing excellent results in the field of compact objects. In this paper we mention a few cases, obtained during the last 2 years (since the spectrograph was mounted on the telescope). But this is only the beginning and we look forward to follow many compact objects for understanding better their physical properties and may be also to discover new kinds of compact objects (and/or systems containing a compact object). 


\section{Acknowledgments}

MCG is supported by the European social fund within the framework of realizing the project "Support of inter-sectoral mobility and quality enhancement of research teams at Czech Technical University in PragueâĂIJ, CZ.1.07/2.3.00/30.0034.

\section{References}

[1] C., Kouveliotou, Magnetars, Proceedings of the National Academy of Science, 96, 5351-5352, (1999)

[2] M., Camenzind, Compact objects in astrophysics, Astronomy and astrophysics library, Berlin: Springer-Verlag, (2007)

[3] N., Gehrels, et al., The Swift Gamma-Ray Burst Mission, ApJ, 611, 1005-1020, (2004)

[4] A., J., Castro-Tirado, et al., The Burst Observer and Optical Transient Exploring System (BOOTES), $A \& A S, 138,583,(1999)$

[5] A., J., Castro-Tirado, et al., Building the BOOTES world-wide Network of Robotic telescopes, ASI Conf. Ser., 7, 313, (2012)

[6] O. Rabaza, et al. Compact low resolution spectrograph (COLORES), Review of Scientific Instruments, 84 (11), 114501, (2014)

[7] M. Jelínek, Study of gamma-ray bursts with robotic telescopes, PhD thesis, U. of Granada, (2014)

[8] M. D., Caballero-Garcia, et al. Initial follow-up of optical transients with COLORES using the BOOTES network, astro-ph/1408.4370, (2014a)

[9] A., Camero-Arranz, et al. Shrinking of the Be disk whilst the X-ray activity increases in the binary system A 0535+262, The Astronomer's Telegram, 7029, (2014b)

[10] A., Camero-Arranz, et al. Detection of a large increase of the size of the Be disk from the X-ray binary A $0535+262$, The Astronomer's Telegram, 6043, (2014a)

[11] F., Giovannelli, et al. Weak optical activity in HDE 245770/A 0535+26 system, The Astronomer's Telegram, 6969, (2015)

[12] M., Nakajima, et al. MAXI/GSC detection of the onset of the outburst from Be/X-ray binary pulsar A 0535+26, The Astronomer's Telegram, 7015, (2015)

[13] A., Camero-Arranz, et al. X-Ray and Optical Observations of A 0535+26, ApJ, 754, 20, (2015)

[14] V. P., Goranskij, et al. Extreme outburst of SS 433 observed in eclipse, The Astronomer's Telegram, 6347, (2014)

[15] S., Charbonnel, et al. Observations of rapid line variations of the Halpha line of SS 433 following a large optical flare, The Astronomer's Telegram, 6355, (2014)

[16] K. V., Sokolovsky, et al. X-ray to radio observations of SS 433 following the bright optical flare, The Astronomer's Telegram, 6364, (2014)

[17] M. D., Caballero-Garcia, et al. Outburst from SS 433 observed with BOOTES-2/COLORES, The Astronomer's Telegram, 6385, (2014b)

[18] Y., Fuchs, et al. SS 433: a phenomenon imitating a Wolf-Rayet star, A\&A, 445, 1041-1052, (2006) 